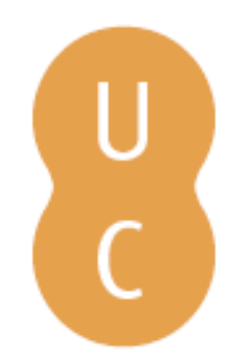

\title{
pommalina
}

\section{Escala de Resiliência para Adultos (ERA)}

$\begin{array}{ll}\text { Autor(es): } & \text { Pereira, Marco; Cardoso, Margarida; Albuquerque, Sara; Janeiro, } \\ \text { Catarina; Alves, Stephanie }\end{array}$

Publicado por: Imprensa da Universidade de Coimbra

URL

persistente: URI:http://hdl.handle.net/10316.2/40586

DOI: $\quad$ DOl:https://doi.org/10.14195/978-989-26-1268-3_2

Accessed : $\quad$ 26-Apr-2023 15:39:32

A navegação consulta e descarregamento dos títulos inseridos nas Bibliotecas Digitais UC Digitalis, UC Pombalina e UC Impactum, pressupõem a aceitação plena e sem reservas dos Termos e Condições de Uso destas Bibliotecas Digitais, disponíveis em https://digitalis.uc.pt/pt-pt/termos.

Conforme exposto nos referidos Termos e Condições de Uso, o descarregamento de títulos de acesso restrito requer uma licença válida de autorização devendo o utilizador aceder ao(s) documento(s) a partir de um endereço de IP da instituição detentora da supramencionada licença.

Ao utilizador é apenas permitido o descarregamento para uso pessoal, pelo que o emprego do(s) título(s) descarregado(s) para outro fim, designadamente comercial, carece de autorização do respetivo autor ou editor da obra.

Na medida em que todas as obras da UC Digitalis se encontram protegidas pelo Código do Direito de Autor e Direitos Conexos e demais legislação aplicável, toda a cópia, parcial ou total, deste documento, nos casos em que é legalmente admitida, deverá conter ou fazer-se acompanhar por este aviso.

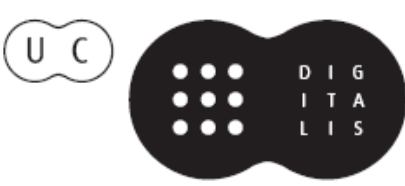


AVALIAÇÃO

$$
\text { FAMILIAR Vorstil Aorracicio }
$$

Q)

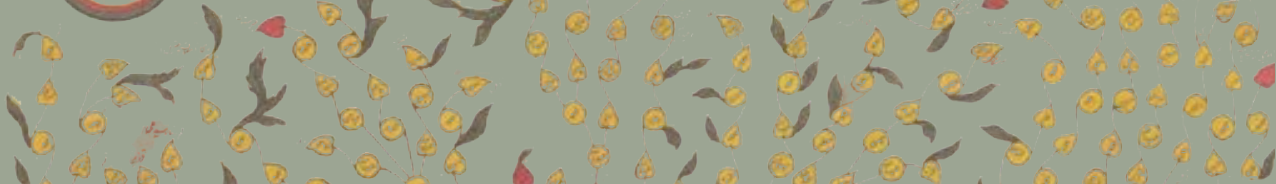

$-1000000$

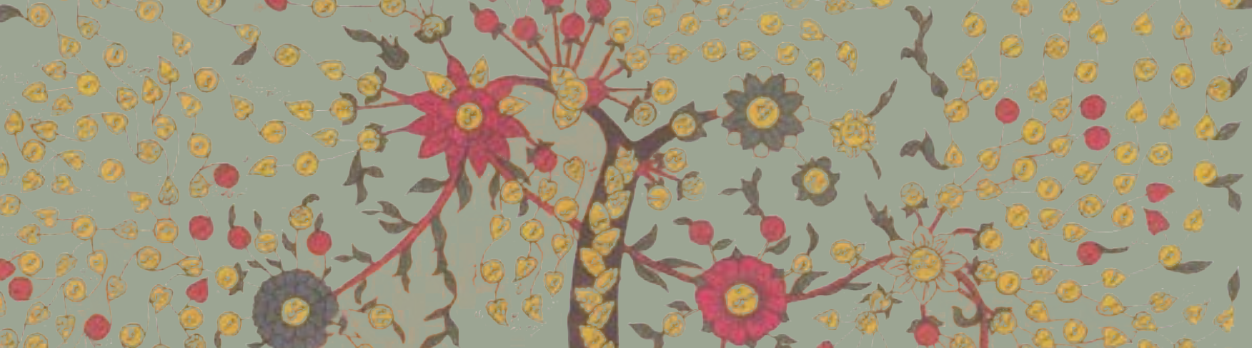

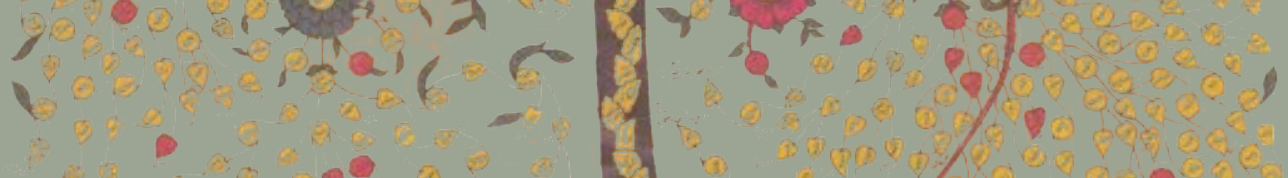

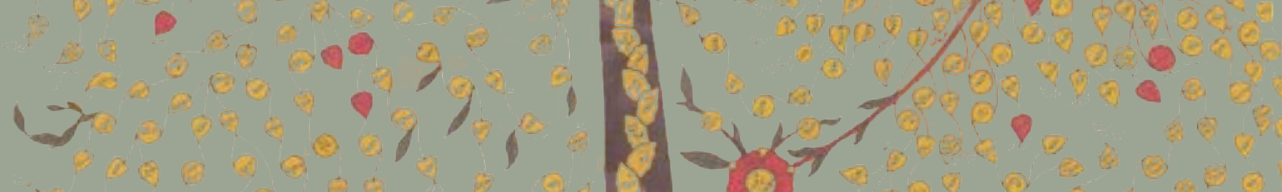

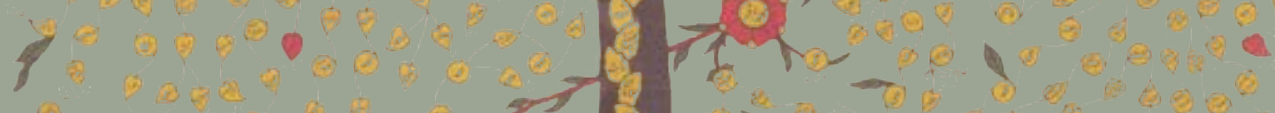

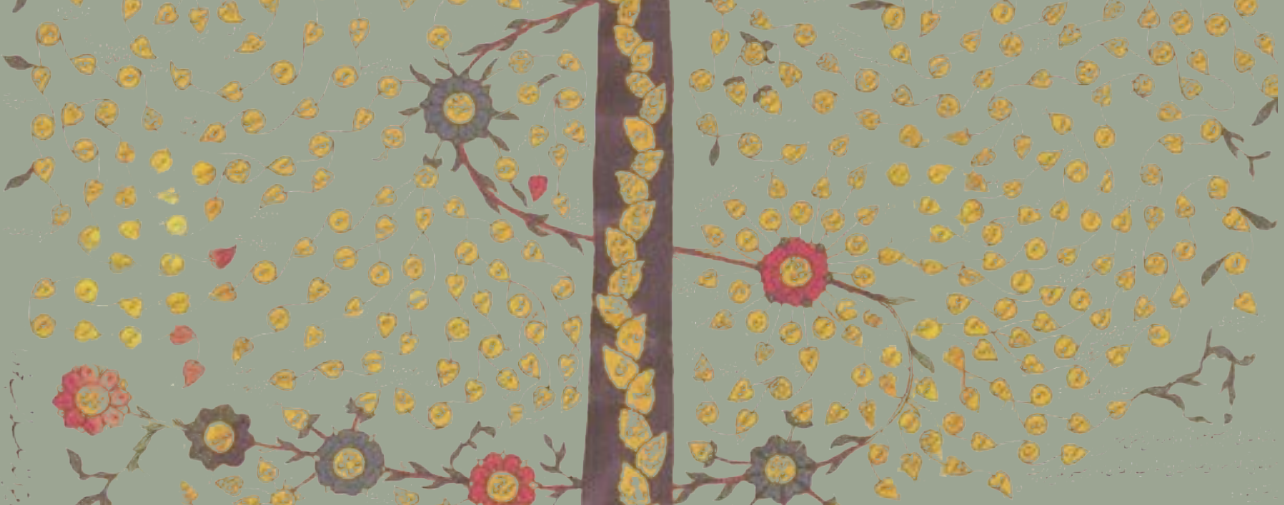

ANA PAULA RELVAS

SOFFA MAJOŔR Wh DE COIMBRA

COORDENAÇÃO 


\section{ESCALA DE RESILIENCIA PARA ADULTOS \\ ( E R A )}

Marco Pereira Margarida Cardoso Sara Albuquerque Catarina Janeiro Stephanie Alves

"Apart from being unlucky enough to get ALS... I have been fortunate in almost every other respect. The help and support I received... have made it possible for me to lead a fairly normal life..."

(Hawking, 1988, p. vii)

\section{Resumo}

Em 2001, Hjemdal, Friborg, Martinussen e Rosenvinge desenvolveram, na Noruega, uma escala para avaliar a resiliência, a Resilience Scale for Adults (RSA). Esta escala foi sofrendo ajustamentos ao longo dos anos, chegando a uma versão final composta por 33 itens organizados em seis fatores. No presente capítulo apresentam-se os estudos de adaptação e validação da versão em Português Europeu da Escala de Resiliência para Adultos (ERA). Numa amostra de validação da população geral $(N=200)$, a análise fatorial exploratória (AFE) sugeriu uma solução fatorial de cinco fatores, não tendo replicado a solução fatorial original. A análise fatorial confirmatória (AFC), numa segunda amostra $(N=304)$, permitiu confirmar o modelo de cinco fatores identificado na AFE, bem como o modelo 
original de seis fatores. O modelo original apresentou, no entanto, melhores índices de ajustamento, pelo que se afigura como válido em Portugal. Neste sentido, na versão em Português Europeu da ERA optou-se pelo modelo original de seis fatores. Este modelo evidenciou indicadores de consistência interna satisfatórios em termos da escala global e da maioria das suas dimensões, apresentando-se como uma escala útil para avaliar os fatores de resiliência. Os resultados obtidos são discutidos com base na utilidade da ERA para a investigação e prática clínica.

Palavras-chave: Escala de Resiliência para Adultos, estudos psicométricos, resiliência.

\section{Abstract}

In 2001, Hjemdal, Friborg, Martinussen, and Rosenvinge developed, in Norway, a questionnaire to assess resilience, the Resilience Scale for Adults (RSA). This scale has been adjusted through the years, and a final version comprising 33 items organized in six factors was achieved. In this chapter we present the adaptation and validation studies of the European Portuguese version of the RSA. In a validation sample of participants of the general population $(N=200)$, the exploratory factor analysis (EFA) suggested a five-factor model, which did not replicate the original factor solution. The confirmatory factor analysis (CFA), in a second sample $(N=304)$, allowed confirming the five-factor model identified in the EFA, as well as the original six-factor model. The original model showed however better adjustment indices and therefore seems to be a valid option in Portugal. Accordingly, in the European Portuguese version of the RSA we opted for the original sixfactor model. This model shows adequate values of internal consistency for the total scale, as well as for most factors, presenting itself as a useful scale to assess resilience factors. The obtained results are discussed in relation to the utility of the RSA for both research and clinical practice.

Keywords: Resilience Scale for Adults, psychometric studies, resilience. 


\section{Instrumento}

\section{O que é, o que avalia e a quem se aplica?}

\section{No Quadro 1 encontra-se a ficha técnica relativa à Escala de Resiliência para}

Adultos (ERA; Friborg, Barlaug, Martinussen, Rosenvinge, \& Hjemdal, 2005;

Friborg, Hjemdal, Rosenvinge, \& Martinussen, 2003; Friborg, Martinussen, \&

Rosenvinge, 2006; Hjemdal, Friborg, Martinussen, \& Rosenvinge, 2001).

Quadro 1.

Ficha técnica da Escala de Resiliência para Adultos (ERA)

A versão portuguesa de 33 itens da Escala de Resiliência para Adultos

O que é? (ERA), no original em inglês Resilience Scale for Adults (RSA), publicada

originalmente em 2001 por O. Hjemdal, O. Friborg, M. Martinussen, e J.

Rosenvinge, na Noruega

A ERA é um questionário de auto-resposta que avalia diversas características de resiliência, contendo itens que na sua estrutura original se organizam nos seis fatores seguintes: Perceção do Self, Planeamento do Futuro, Competências Sociais, Coesão Familiar, Recursos Sociais e Estilo Estruturado

\begin{tabular}{lcl}
\hline & \multicolumn{2}{c}{ Estrutura da ERA } \\
\hline Subescala & Número Itens & Descrição \\
\hline $\begin{array}{l}\text { Perceção do Self } \\
\text { (PS) }\end{array}$ & 6 & $\begin{array}{l}\text { Avalia a confiança nas próprias } \\
\text { capacidades e julgamentos, auto- } \\
\text { eficácia e expetativas realistas }\end{array}$ \\
\hline $\begin{array}{l}\text { Planeamento do } \\
\text { Futuro }\end{array}$ & 4 & $\begin{array}{l}\text { Avalia a capacidade de planeamento } \\
\text { antecipado, de ter uma visão otimista } \\
\text { e de se orientar por objetivos claros e } \\
\text { alcançáveis }\end{array}$ \\
\hline
\end{tabular}

Competências

Sociais

(CS)

O que avalia?

Estilo Estruturado

(EE)

(CF)

Recursos Sociais

(RS)
Avalia a flexibilidade em interações sociais, a capacidade de criar novas amizades, sentir-se à vontade em ambientes sociais e o uso positivo do humor

Avalia a capacidade de ter e seguir rotinas, de organização do próprio tempo, e a preferência por objetivos e planos claros antes da realização das atividades

Avalia se os valores são partilhados ou discordantes na família, se os membros da família apreciam passar tempo juntos, se têm uma visão otimista do futuro, se são leais uns com os outros e se têm um sentimento de apreciação e apoio mútuo

Mede a disponibilidade de apoio social, se existe uma pessoa confidente fora do núcleo familiar (como amigos ou outros que os apreciam e encorajam) e se existem pessoas a quem recorrer fora da família caso necessitem de ajuda 


\section{Fundamentação e bistória}

No âmbito da tendência recente de mudança de uma perspetiva patogénica (i.e., focada na origem da doença) para uma perspetiva salutogénica (i.e., focada na origem da saúde), que enfatiza a saúde, o bem-estar e a qualidade de vida, bem como as competências positivas dos sujeitos, o conceito de resiliência tem vindo a adquirir cada vez maior atenção e relevância. A investigação sobre resiliência tem crescido nas últimas décadas, em particular em domínios do conhecimento como a Psicologia do Desenvolvimento, a Psicologia da Família e a Psicologia Clínica e da Saúde (e.g., Becvar, 2007; Davydov, Stewart, Ritchie, \& Chaudieu, 2010; Tedeschi \& Kilmer, 2005; Walsh, 2003). O foco crescente no papel da resiliência na promoção da saúde representa uma abordagem mais integradora à prevenção e tratamento, dado que permite equilibrar a ênfase na patologia e na vulnerabilidade com o foco nos mecanismos protetores e na adaptação positiva.

Do ponto de vista concetual, a resiliência tem vindo a ser definida a partir de duas perspetivas distintas: (1) a resiliência enquanto resultado, definida como o funcionamento adaptativo após a vivência de uma situação de adversidade; e (2) a resiliência enquanto recurso, como fator de proteção em situações indutoras de stress, potenciando a adaptação a estas situações (Schaap, Galen, Ruijter, \& Smeets, 2009). No âmbito do primeiro ponto, a resiliência é assim definida como a capacidade de adaptação, competência ou funcionamento positivo dos indivíduos, quando na presença de adversidade ou risco (Masten, 2007), sendo que a existência de risco é um aspeto fundamental deste conceito. Para Hjemdal, Friborg, 
Stiles, Rosenvinge e Martinussen (2006), o problema da concetualização da resiliência como resultado é que esta definição deixa muito pouco espaço para a previsão. Por outras palavras, define o resultado final, mas não o que contribui para esse resultado.

Para incluir esta perspetiva preditiva e facilitar a investigação sobre processos, Hjemdal, Friborg, Stiles, Rosenvinge e Martinussen (2006) definem a resiliência como os fatores protetores, processos e mecanismos que contribuem para um resultado positivo, apesar da experiência de acontecimentos que incorrem de um risco significativo de desenvolvimento de psicopatologia. A resiliência é, neste sentido, entendida como um constructo multidimensional, onde estão implícitas não só competências psicológicas, mas também a capacidade dos indivíduos recorrerem aos diversos sistemas do seu contexto (e.g., família, rede social) para melhor lidar com situações de stress e de adversidade. $\mathrm{Na}$ linha desta segunda perspetiva, a resiliência é assim entendida como um conjunto de traços de personalidade, qualidades e competências individuais, relativamente estáveis, que estão associadas à capacidade de superar e ultrapassar a adversidade e lidar com o stress (Connor \& Davidson, 2003; Smith-Osborne \& Bolton, 2013). As pessoas resilientes são vistas como mais flexíveis que as pessoas vulneráveis e lidam com as situações de stress ou adversidades usando diversos recursos protetores, quer individuais quer interpessoais e contextuais (Friborg et al., 2003). A este respeito, é de salientar que a resiliência não protege os indivíduos dos acontecimentos de vida adversos. Na realidade, os indivíduos resilientes podem sentir perturbações transitórias no funcionamento normal, porém, estes indivíduos mostram uma capacidade de lidar de forma mais funcional e flexível com esses acontecimentos, exibindo deste modo uma trajetória estável de funcionamento saudável ao longo do tempo (Bonanno, 2004).

A avaliação da resiliência tem sido realizada de modos muito distintos, dada a multiplicidade e complexidade que lhe está subjacente (Curtis \& Cicchetti, 2007). Com efeito, a dificuldade em definir resiliência tem sido bastante reconhecida (Luthar, Cicchetti, \& Becker, 2000; Masten, 2007), o que tem criado desafios consideráveis na sua operacionalização. Apesar 
de alguma controvérsia em torno do uso de medidas de auto-resposta na avaliação da resiliência (Reppold, Mayer, Almeida, \& Hutz, 2012), têm havido várias tentativas para a sua avaliação. No entanto, são ainda escassas as medidas validadas para a avaliação da resiliência, havendo também pouco consenso sobre quais são as melhores para a avaliar e descrever (Hoge, Austin, \& Pollack, 2007). A maioria das medidas está ligada apenas em parte a este conceito (i.e., avaliando, por exemplo, a robustez e o stress percebido) e é aplicada a populações específicas, o que leva a que seja difícil uma análise e comparação de resultados (Connor \& Davidson, 2003; Friborg et al., 2005; Schaap et al., 2009). A necessidade de instrumentos válidos de avaliação da resiliência que sejam fáceis de aplicar e interpretar é, portanto, evidente (Connor \& Davidson, 2003; Vaishnavi, Connor, \& Davidson, 2007), pois sem uma compreensão da multidimensionalidade e dinâmica deste conceito será difícil entender a disfuncionalidade e as dificuldades de adaptação (Bonanno, 2004).

$\mathrm{Na}$ avaliação da resiliência, quer em adolescentes quer em adultos, têm sido desenvolvidas, no entanto, algumas escalas (Ahern, Kiehl, Sole, \& Byers, 2006), das quais se destacam as seguintes: Resilience Scale (RS; Wagnild \& Young, 1993); Ego-Resiliency Scale (ER89; Bock \& Kremen, 1996); Connor-Davidson Resilience Scale (CD-RISC; Connor \& Davidson, 2003), assim como as suas versões abreviadas (CD-RISC10; Campbell-Sills \& Stein, 2007; CD-RISC2; Vaishnavi et al., 2007); Adolescent Resilience Scale (ARS; Oshio, Kaneko, Nagamine, \& Nakaya, 2003); Resiliency Scales for Children \& Adolescents (RSCA; Prince-Embury, 2008); Resilience Scale for Adolescents (READ; Hjemdal, Friborg, Stiles, Martinussen, \& Rosenvinge, 2006); Brief-Resilient Coping Scale (BRCS; Sinclair \& Wallston, 2004); e Brief Resilience Scale (BRS; Smith et al., 2008). A importância da avaliação da resiliência é de tal modo central que nos últimos anos têm surgido diversas revisões que procuraram sintetizar informação sobre os instrumentos (e respetiva qualidade) atualmente disponíveis (para revisão cf. Ahern et al., 2006; Gurgel, Plentz, Joly, \& Reppold, 2013; Smith-Osborne \& Bolton, 2013; Windle, Bennett, \& Noyes, 2011).

Para além das medidas enunciadas, na Noruega, foi desenvolvida uma nova escala de avaliação de resiliência, que foi denominada de Escala 
de Resiliência para Adultos (ERA; Friborg et al., 2003; 2005; Hjemdal et al., 2001). Esta escala foi desenvolvida com o objetivo de colmatar a escassez de medidas de avaliação da resiliência, nomeadamente na população adulta, mas também com um propósito de capturar um conjunto de fatores protetores fundamentais (Hjemdal et al., 2001). Do ponto de vista concetual, no desenvolvimento da ERA, os autores tiveram em consideração os três grandes grupos de recursos protetores descritos na literatura (Cicchetti \& Garmezy, 1993; Masten \& Coatsworth, 1998; Rutter, 1990): (1) competências psicológicas/disposicionais e atributos do indivíduo; (2) apoio familiar e ambiente familiar coeso; e (3) presença de sistemas de apoio externo que promovem um coping eficiente e o ajustamento.

A versão inicial da ERA continha 45 itens (resultantes de uma poll de 295 itens), estando organizada em cinco fatores: Competência Pessoal, Competência Social, Suporte Social, Coesão Familiar e Estrutura Pessoal (Hjemdal et al., 2001). Em 2003, com vista a um novo afinamento da escala, Firborg e colaboradores adicionaram novos itens, sujeitando a ERA a novas análises. Este procedimento levou a uma versão composta por 37 itens. Numa revisão posterior, na qual os autores reformularam os itens e alteraram a escala de resposta de tipo Likert para um formato de resposta de diferencial semântico (e.g., para o item 16, "A minha família caracteriza-se por...", a escala de sete pontos variou entre "desunião" e "coesão saudável"), de modo a reduzir a tendência para a aquiescência, chegou-se à versão final atual de 33 itens (Friborg et al., 2005). Posteriormente, Hjemdal, Friborg, Stiles, Rosenvinge e Martinussen (2006), e após novas análises fatoriais confirmatórias, sugeriram uma estrutura em seis fatores: Perceção do Self, Planeamento do Futuro, Competências Sociais, Estilo Estruturado, Coesão Familiar e Recursos Sociais. Em termos globais, o que distingue as duas estruturas é que a dimensão competências pessoais encontra-se dividida nos fatores Perceção do Self e Planeamento do Futuro. Na sua versão final, a ERA é composta assim por 33 itens e permite avaliar os recursos protetores que promovem a resiliência na idade adulta (Hjemdal, Friborg, Stiles, Rosenvinge, \& Martinussen, 2006). Os autores construíram assim um modelo da resiliência que mostrou ser ajustado 
nos vários estudos psicométricos realizados e que abrange fatores que estão incluídos num modelo global que compreende a resiliência como um conceito multidimensional relacionado com as três grandes categorias de recursos protetores anteriormente enunciadas.

Para além das aplicações da ERA em diferentes amostras da Noruega (e.g., Friborg et al., 2003, 2005, 2006; Hjemdal, Friborg, Stiles, Rosenvinge, \& Martinussen, 2006), encontram-se disponíveis uma versão Persa desenvolvida em conjunto com investigadores do Irão (Jowkar, Friborg, \& Hjemdal, 2010), uma versão em Francês desenvolvida na Bélgica (Hjemdal et al., 2011) e uma versão em Português do Brasil (Hjemdal, Roazzi, Dias, Roazzi, \& Vikan, 2009). Nestas diferentes aplicações da ERA, todas as versões permitiram confirmar a estrutura fatorial proposta, ainda que os valores de consistência interna tenham diferido entre estudos. Nomeadamente, foram obtidos valores do alfa de Cronbach entre .76 e .84 (.90 para a escala total) no Irão (Jowkar et al., 2010), entre .63 e .78 (.84 para o total da escala) na Bélgica (Hjemdal et al., 2011) e entre .56 e .79 no Brasil (Hjemdal et al., 2009).

Os estudos psicométricos preliminares da versão em Português Europeu da ERA foram apresentados e publicados recentemente (Pereira, Cardoso, Alves, Narciso, \& Canavarro, 2013), mas não foram realizados estudos confirmatórios da estrutura da ERA. No presente capítulo, apresentam-se assim os estudos de adaptação e validação da versão em Português Europeu da ERA e analisa-se se a estrutura fatorial original é replicada em Portugal.

\section{Estudos em Portugal}

\section{Como foi desenvolvido/adaptado e validado?}

\section{Estudos de tradução e adaptação}

Os estudos iniciais de desenvolvimento e validação da Escala de Resiliência para Adultos em Portugal resultam de um processo, decorrido entre outubro de 2009 e maio de 2010, no âmbito de um projeto de Pós-Doutoramento em 
curso na Faculdade de Psicologia e de Ciências da Educação da Universidade de Coimbra.

O primeiro passo deste processo consistiu na obtenção da autorização dos autores da ERA. Em seguida, iniciou-se o processo de tradução do instrumento para Português Europeu. Após contacto com os autores da escala original (O. Friborg e O. Hjemdal), tomou-se conhecimento de que já existia uma versão em Português (do Brasil). Dada a existência de divergências linguísticas entre o Português Europeu e do Brasil, optou-se por realizar o processo de tradução completo. A ERA foi inicialmente traduzida pelo investigador responsável pela validação da escala em Portugal e, posteriormente, esta versão foi revista por outros três investigadores com formação em Psicologia. Depois de incorporadas as sugestões dos três "revisores", chegou-se a uma tradução conciliadora que foi depois submetida a retroversão por uma tradutora independente fluente em Português e Inglês. A versão que resultou da retroversão foi comparada com a versão inicial e, em conjunto com a tradutora, foram feitos os ajustamentos necessários à primeira versão traduzida.

Concluídos os procedimentos de tradução e adaptação da ERA, iniciou-se o estudo de campo quantitativo. No estudo de validação, a ERA foi administrada a uma amostra composta por 200 participantes da população geral. A recolha da amostra (por conveniência) foi realizada junto da população geral, bem como de estudantes do Ensino Superior da Universidade de Coimbra. Num total de 248 protocolos distribuídos, 204 foram preenchidos e devolvidos (taxa de resposta $=82.3 \%$ ), tendo quatro sido excluídos por preenchimento indevido. Cerca de um mês após a primeira aplicação, foi solicitado a 60 indivíduos que preenchessem uma segunda aplicação da ERA, de modo a avaliar a estabilidade temporal. Dos 60 participantes contactados, foi recebido um total de 45 protocolos de avaliação (taxa de resposta $=75 \%$ ).

Para além da ERA, a bateria de avaliação do protocolo de validação incluiu um questionário de dados sociodemográficos e relativos à história relacional e familiar e acontecimentos de vida, bem como os instrumentos de auto-resposta seguintes: Connor-Davidson Resilience Scale (CD-RISC; Connor \& Davidson, 2003; Versão Portuguesa (VP): Faria \& Ribeiro, estudos em curso); Escala de Auto-Eficácia Geral Percepcionada (EAEGP; 
Schwarzer \& Jerusalem, 1995); Perceived Stress Scale (PSS; Cohen, Kamark \& Mermelstein, 1983; VP: Mota-Cardoso Araújo, Ramos, Gonçalves, \& Ramos, 2002); e o instrumento de avaliação da qualidade de vida, World Health Organization Quality of Life - versão abreviada (WHOQOL-Bref; WHOQOL Group, 1998; VP: Vaz Serra et al., 2006).

A amostra dos estudos de validação foi constituída, como referido, por 200 participantes, maioritariamente do sexo feminino ( $n=106 ; 53 \%)$ e com uma idade média de 35.20 anos $(D P=14.94$; amplitude: 18-72). A média de anos de escolaridade foi de 14.09 anos $(D P=3.36)$. Nas restantes variáveis, observou-se uma distribuição similar em termos de estado civil ( $51 \%$ casados/unidos de facto e 48\% solteiros) e situação profissional (49\% estudantes e $47.5 \%$ empregados). Em relação ao meio de proveniência, a maioria dos participantes residia em meio urbano ( $n=146 ; 73 \%$ ).

No âmbito do referido projeto de Pós-Doutoramento, uma amostra adicional de 304 participantes preencheu a ERA. Esta amostra integrou 152 casais da população geral, que responderam negativamente à presença de uma situação de particular adversidade, e casais em contexto de adversidade (e.g., diagnóstico de anomalia congénita de um filho, doença psiquiátrica de um dos elementos do casal). Os procedimentos de recolha destes grupos encontram-se descritos com detalhe em outras publicações dos autores (Albuquerque, Pereira, Fonseca, \& Canavarro, 2012; Alves, Pereira, Janeiro, Narciso, \& Canavarro, 2014).

Esta segunda amostra foi assim composta por 304 participantes, com igual proporção de homens e mulheres e com uma média de idades de 42.43 anos $(D P=10.46$; amplitude: 23-76). A média de anos de escolaridade foi de 10.99 anos $(D P=4.04)$. Os participantes eram na maioria casados (84.2\%) e estavam em situação de emprego (82.1\%). Em relação ao meio de proveniência, a maioria dos participantes residia em meio urbano (91.7\%).

\section{Estudos descritivos}

A análise descritiva dos 33 itens da ERA encontra-se no Quadro 2, onde se apresentam os valores para a média $(M)$, desvio-padrão $(D P)$, moda, 
amplitude, assimetria e curtose. São também apresentadas as análises de consistência interna, nomeadamente os valores das correlações item-total e os valores do coeficiente alfa de Cronbach quando o item é excluído.

Quadro 2.

Estatísticas descritivas dos itens da ERA e consistência interna

\begin{tabular}{|c|c|c|c|c|c|c|c|c|}
\hline Item & $M$ & $D P$ & Moda & Amplitude & Assimetria & Curtose & $\begin{array}{c}\text { Correlação } \\
\text { Item-Total } \\
\text { Corrigida }\end{array}$ & $\begin{array}{l}\text { Alfa com } \\
\text { Item } \\
\text { Excluído }\end{array}$ \\
\hline 1 & 5.17 & 1.34 & 6 & $1-7$ & -0.62 & -0.18 & .48 & .901 \\
\hline 2 & 5.52 & 1.28 & 6 & $2-7$ & -0.98 & 0.76 & .52 & .900 \\
\hline 3 & 4.92 & 1.70 & 6 & $1-7$ & -0.59 & -0.44 & .28 & .905 \\
\hline 4 & 5.20 & 1.53 & 6 & $1-7$ & -0.89 & 0.20 & .41 & .902 \\
\hline 5 & 5.91 & 1.27 & 7 & $1-7$ & -1.56 & 2.69 & .44 & .901 \\
\hline 6 & 4.51 & 1.77 & 6 & $1-7$ & -0.41 & -0.76 & .18 & .907 \\
\hline 7 & 5.42 & 1.31 & 6 & $1-7$ & -0.77 & -0.01 & .56 & .900 \\
\hline 8 & 4.50 & 1.55 & 4 & $1-7$ & -0.44 & -0.43 & .45 & .901 \\
\hline 9 & 5.33 & 1.35 & 6 & $1-7$ & -0.73 & 0.41 & .36 & .903 \\
\hline 10 & 5.84 & 1.46 & 7 & $1-7$ & -1.60 & 2.18 & .53 & .900 \\
\hline 11 & 6.08 & 1.16 & 7 & $1-7$ & -1.76 & 3.42 & .63 & .899 \\
\hline 12 & 4.42 & 1.58 & 5 & $1-7$ & -0.20 & -0.67 & .18 & .906 \\
\hline 13 & 5.23 & 1.32 & 6 & $1-7$ & -0.71 & 0.17 & .45 & .901 \\
\hline 14 & 5.10 & 1.40 & 6 & $1-7$ & -0.72 & -0.05 & .56 & .899 \\
\hline 15 & 4.77 & 1.74 & 6 & $1-7$ & -0.63 & -0.47 & .37 & .903 \\
\hline 16 & 5.68 & 1.41 & 7 & $1-7$ & -1.18 & 1.04 & .49 & .901 \\
\hline 17 & 5.76 & 1.13 & 6 & $2-7$ & -0.95 & 0.65 & .44 & .901 \\
\hline 18 & 4.65 & 1.65 & 6 & $1-7$ & -0.47 & -0.57 & .32 & .904 \\
\hline 19 & 5.47 & 1.39 & 6 & $1-7$ & -1.06 & 0.84 & .59 & .899 \\
\hline 20 & 5.13 & 1.43 & 6 & $1-7$ & -0.73 & 0.07 & .64 & .898 \\
\hline 21 & 4.94 & 1.57 & 6 & $1-7$ & -0.67 & -0.24 & .47 & .901 \\
\hline 22 & 5.17 & 1.38 & 6 & $1-7$ & -0.68 & -0.30 & .50 & .900 \\
\hline 23 & 5.43 & 1.39 & 6 & $1-7$ & -0.77 & -0.07 & .52 & .900 \\
\hline 24 & 5.14 & 1.35 & 6 & $1-7$ & -0.53 & -0.31 & .30 & .903 \\
\hline 25 & 4.51 & 1.70 & 6 & $1-7$ & -0.41 & -0.93 & .52 & .900 \\
\hline 26 & 5.78 & 1.20 & 6 & $1-7$ & -1.23 & 1.56 & .56 & .900 \\
\hline 27 & 5.72 & 1.31 & 6 & $1-7$ & -1.33 & 1.70 & .45 & .901 \\
\hline 28 & 6.04 & 1.27 & 7 & $1-7$ & -1.79 & 3.19 & .51 & .900 \\
\hline 29 & 4.56 & 1.59 & 6 & $1-7$ & -0.45 & -0.59 & .33 & .903 \\
\hline 30 & 5.17 & 1.52 & 6 & $1-7$ & -0.70 & -0.17 & .45 & .901 \\
\hline 31 & 5.10 & 1.51 & 6 & $1-7$ & -0.46 & -0.77 & .54 & .900 \\
\hline 32 & 5.67 & 1.43 & 7 & $1-7$ & -1.27 & 1.37 & .43 & .901 \\
\hline 33 & 5.70 & 1.17 & 6 & $2-7$ & -1.03 & 0.74 & .66 & .898 \\
\hline
\end{tabular}


Os resultados encontrados indicam que o item da ERA que apresenta uma média mais elevada $(M=6.08 ; D P=1.16)$ corresponde ao item 11 "Aqueles que são bons a encorajar-me... são alguns amigos próximos/ familiares". Por outro lado, o item 12 "Quando inicio novas coisas/ projectos... prefiro ter um plano minucioso" obteve a média mais baixa $(M=4.42 ; D P=1.58)$. De um modo geral, os valores oscilam entre 1 e 7 , sendo que o valor mais frequente é o 6 . Os valores mínimos e máximos registados nas respostas a cada item ilustram que, em 30 dos 33 itens, cada uma das alternativas foi escolhida por pelo menos um participante. Quanto à assimetria, os itens apresentam todos um valor negativo, com destaque para um maior afastamento dos itens 5, 10, $11,16,19,26,27,28,32$ e 33 (assimetria $>1.00$ e $<1.79$ ). Em termos do grau de achatamento da distribuição, os itens que se encontram mais afastados do valor zero são os itens 5, 10, 11, 16, 26, 27, 28, e 32, sendo que os valores para estes oito itens são todos positivos (curtose $>1.00 \mathrm{e}<3.42)$. Resultados semelhantes foram obtidos em estudos anteriores da ERA, suportando que a não-normalidade nos resultados de resiliência é um fenómeno comum.

\section{Estudos de precisão}

Para averiguar a precisão dos dados, procedemos à análise da consistência interna dos itens da ERA, através do cálculo do coeficiente alfa de Cronbach, da escala total e dos fatores que a compõem. Assim, para o total dos itens, a ERA apresentou um alfa de Cronbach de .90. O valor do coeficiente Split-Half foi de .90. A análise dos valores do coeficiente alfa da escala total aquando a exclusão de qualquer um dos itens mostrou que a sua exclusão não aumenta de forma expressiva a consistência interna total da escala (cf. Quadro 2), com exceção dos casos em que são excluídos os itens 6 e 12. Os valores de correlação item-total (corrigida) são, na generalidade, aceitáveis, variando entre .18 e .66 . Em relação à estabilidade temporal, verificou-se que as correlações entre os itens foram todas superiores a .60 e estatisticamente 
significativas para um nível de significação < .001; a exceção foi o item $10(r=.31, p=.038)$.

\section{Estudos de validade de constructo: Análise fatorial exploratória}

A validade de constructo da ERA foi verificada através da análise fatorial exploratória (AFE) na amostra de validação inicial. Uma primeira AFE, seguida de rotação Promax, culminou com uma solução de oito fatores, cuja variância total explicada era de $60.0 \%$. Uma vez que os três últimos fatores explicavam uma percentagem da variância relativamente baixa (i.e., respetivamente $3.7 \%, 3.5 \%$ e $3.2 \%$ ), aliado ao facto destes últimos conterem poucos itens, optou-se por pedir a extração de seis fatores, de acordo com a estrutura original da escala.

$\mathrm{Na}$ segunda solução, a variância cumulativa explicada foi de $53.4 \%$. O Fator 1 explicava $26.9 \%$ da variância, o Fator 2 explicava $7.3 \%$, o Fator 3 explicava $6.4 \%$, o Fator 4 explicava $4.9 \%$, o Fator 5 explicava $4.2 \%$ e o Fator 6 explicava 3,7\% da variância. O teste de Keiser-Meyer-Olkin (KMO), com o resultado de .86 e com o teste de esfericidade de Bartlett $(p<.001)$ suportam a utilização desta análise.

Uma vez que no Fator 6 saturavam apenas dois itens (i.e., itens 6 e 8), optou-se por alocar o item 8 no Fator 1, onde saturava também de forma satisfatória e optou-se por eliminar o item 6 , pois este não saturava em nenhum dos restantes fatores. De assinalar que este item foi o que mais fez inflacionar o valor do alfa de Cronbach quando excluído. A realização de nova análise fatorial apenas com os 32 itens, e forçada a cinco fatores, revelou a estrutura apresentada no Quadro 3. Assim, na solução de cinco fatores, o Fator 1 foi designado de Competências Pessoais; o Fator 2 foi nomeado de Coesão Familiar; o Fator 3 recebeu a designação de Recursos Sociais; o Fator 4 de Competências Sociais e o Fator 5 foi designado de Estilo Estruturado. A denominação dos fatores seguiu a designação dada pelos autores originais da ERA e, na sua globalidade, a estrutura resultante da AFE apresenta semelhanças com a estrutura inicialmente proposta. 
Quadro 3.

Matriz rodada, comunalidades e variância explicada (Rotação Promax) - ERA

\begin{tabular}{|c|c|c|c|c|c|}
\hline \multirow{2}{*}{ Itens } & \multicolumn{5}{|c|}{ Fatores } \\
\hline & 1 & 2 & 3 & 4 & 5 \\
\hline 13. Os meus juízos e decisões... & .79 & & & & \\
\hline 29. Acontecimentos na vida que não consigo influenciar... & .77 & & & & \\
\hline 1. Quando acontece alguma coisa imprevista... & .73 & & & & \\
\hline 14. Os meus objectivos... & .72 & & & & \\
\hline 25. Em períodos difíceis tenho tendência... & .69 & & & & \\
\hline 7. Os meus problemas pessoais... & .62 & & & & \\
\hline 2. Os meus planos para o futuro... & .61 & & & & \\
\hline 19. Acreditar em mim... & .43 & & & & \\
\hline 8. Sinto que o meu futuro parece... & .39 & & & & \\
\hline 16. A minha família caracteriza-se por... & & .86 & & & \\
\hline 31. Na minha família, gostamos de... & & .83 & & & \\
\hline 27. Perante outras pessoas, a nossa família mostra... & & .68 & & & \\
\hline 22. Em períodos difíceis, a minha família... & & .58 & & & \\
\hline 4. Perspectiva da família sobre o importante na vida... & & .56 & & & \\
\hline 10. Eu sinto-me [muito feliz/infeliz na família]... & & .56 & & & \\
\hline 23. Quando um familiar passa por uma crise/emergência... & & .49 & & & \\
\hline 18. Sou bom (boa) a... & & .49 & & & \\
\hline 28. Eu recebo apoio de... & & & .85 & & \\
\hline 5. Posso discutir assuntos pessoais com... & & & .73 & & \\
\hline 11. Aqueles que são bons a encorajar-me... & & & .65 & & \\
\hline 32. Quando preciso [ajuda]... & & & .56 & & \\
\hline 17. A ligação entre os meus amigos é... & & & .41 & & \\
\hline 33. Os meus amigos/familiares próximos ${ }^{\mathrm{a}} \ldots$ & & & .30 & & \\
\hline 15. Novas amizades são algo... & & & & .85 & \\
\hline 21. Conhecer novas pessoas... & & & & .84 & \\
\hline 3. Eu gosto de estar... & & & & .57 & \\
\hline 30. Para mim, pensar em bons tópicos de conversa... & & & & .51 & \\
\hline 26. Quando estou com outras pessoas ${ }^{\mathrm{a}} \ldots$ & & & & .24 & \\
\hline 12. Quando inicio novas coisas/projectos... & & & & & .77 \\
\hline 24. Regras e rotinas habituais... & & & & & .70 \\
\hline 9. Ser flexível em contextos sociais... & & & & & .53 \\
\hline 20. Os meus objectivos para o futuro... & & & & & .46 \\
\hline
\end{tabular}

F1: Competências Pessoais; F2: Coesão Familiar; F3: Recursos Sociais; F4: Competências Sociais; e F5: Estilo Estruturado.

${ }^{\text {a }}$ Os itens 26 e 30 saturaram igualmente nos Fatores 2 e 4, respetivamente. Por razões concetuais e após análise das duas matrizes resultantes da AFE, optou-se por mudar os itens para os fatores onde se encontram nesta solução final (respetivamente, Fator 4 e 3).

Considerando esta solução fatorial, os alfas de Cronbach variaram entre .61 (F5: Estilo Estruturado) e .84 (F1: Competências Pessoais). Os valores da correlação teste-reteste, tendo variado entre .79 (F2: Coesão Familiar) e .93 (F1: Competências Pessoais), atestam a estabilidade temporal do instrumento. 


\section{Estudos de validade}

Os fatores da ERA encontram-se significativamente associados entre si, o que permite admitir a existência da validade de conteúdo desta escala. As correlações são maioritariamente moderadas, com uma variação entre .33 (entre os fatores Coesão Familiar e Competências Sociais) e .59 (entre os fatores Coesão Familiar e Recursos Sociais).

Com a finalidade de avaliar as validades concorrente e divergente, foram analisadas as correlações entre os fatores da ERA com outros instrumentos. A validade concorrente foi avaliada através da correlação entre os fatores da ERA e os da CD-RISC (Quadro 4). As correlações variaram entre .07 (entre o Fator 3 da CD-RISC e o fator Estilo Estruturado da ERA) e .67 (entre o Fator 1 da CD-RISC e o fator Competências Pessoais). As associações mais baixas dos fatores da ERA registaram-se com o fator Influências Espirituais da CD-RISC (F5).

Quadro 4 .

Validade concorrente (correlação entre a ERA e a CD-RISC)

\begin{tabular}{|c|c|c|c|c|c|}
\hline \multirow{2}{*}{ Fatores da ERA } & \multicolumn{5}{|c|}{ Fatores da CD-RISC } \\
\hline & F1 & F2 & F3 & F4 & F5 \\
\hline Competências Pessoais & $.67^{* * * *}$ & $.46^{\text {**** }}$ & $.39^{\text {**** }}$ & $.34^{* * * *}$ & $.39^{* * * * *}$ \\
\hline Coesão Familiar & $.46^{* * * *}$ & $.22^{* *}$ & .10 & $.23^{* *}$ & $.27^{* * * *}$ \\
\hline Recursos Sociais & $.62^{* * * * *}$ & $.41^{* * * *}$ & $.30^{* * *}$ & $.25^{* * *}$ & $.36^{* * * *}$ \\
\hline Competências Sociais & $.40^{* * * * *}$ & $.37^{* * * *}$ & $.31^{* * * *}$ & $.21^{* *}$ & $.28^{* * * * *}$ \\
\hline Estilo Estruturado & .13 & $.21^{* *}$ & .07 & $.15^{*}$ & $.21^{* *}$ \\
\hline
\end{tabular}

F1: Perceção de competência pessoal, padrões elevados e tenacidade; F2: Confiança nos instintos, tolerância às emoções negativas e fortalecimento dos efeitos do stress; F3: Aceitação positiva da mudança e relações interpessoais seguras; F4: Controlo; e F5: Influências espirituais.

$* p<.05 ; * * * 201 ; * * * 0<.001$

A validade divergente da ERA foi avaliada através de correlações com as seguintes escalas: EAGP (auto-eficácia), PSS (perceção de stress) e WHOQOL-Bref (qualidade de vida). Como se pode verificar, a correlação entre os fatores da ERA foi positiva com a autoeficácia percecionada e a perceção de qualidade de vida e negativa com a perceção de stress (cf. Quadro 5). 
Quadro 5.

Validade divergente da ERA

\begin{tabular}{|c|c|c|c|c|c|}
\hline \multirow{2}{*}{ Escalas } & \multicolumn{5}{|c|}{ Fatores da ERA } \\
\hline & F1 & F2 & F3 & F4 & F5 \\
\hline EAEGP (total) & $.64^{* * * *}$ & $.32^{\text {***** }}$ & $.28^{* * * * *}$ & $.28^{* * * *}$ & $.29^{* * * *}$ \\
\hline PSS (total) & $-.60^{* * * *}$ & $-.28^{* * * * *}$ & $-.26^{* * * *}$ & $-.31^{* * * *}$ & $-.25^{* * * *}$ \\
\hline \multicolumn{6}{|l|}{ WHOQOL-Bref } \\
\hline Físico & $.42^{* * * *}$ & $.40^{* * * * *}$ & $.25^{* * * * *}$ & $.18^{* *}$ & $.19^{* *}$ \\
\hline Psicológico & $.67^{* * * *}$ & $.52^{* * * *}$ & $.35^{* * * * *}$ & $.33^{* * * *}$ & $.30^{* * *}$ \\
\hline Relações Sociais & $.46^{* * * *}$ & $.39^{* * * * *}$ & $.42^{* * * * *}$ & $.27^{* * * *}$ & $.30^{* * * *}$ \\
\hline Ambiente & $.51^{* * * *}$ & $.43^{* * * *}$ & $.30^{* * * * * *}$ & $.23^{* * *}$ & $.23^{* *}$ \\
\hline Faceta Geral & $.30^{* * * *}$ & $.37^{* * * * *}$ & $.24^{* * *}$ & .10 & $.16^{*}$ \\
\hline
\end{tabular}

F1: Competências Pessoais; F2: Coesão Familiar; F3: Recursos Sociais; F4: Competências Sociais; e F5: Estilo Estruturado.

$* p<.05 ; * * * 2.01 ; * * * 0<.001$

\section{Estudos de validade de constructo: Análise fatorial confirmatória}

Na segunda amostra foi realizada uma análise fatorial confirmatória (AFC) com o objetivo de testar o modelo unidimensional, o modelo de cinco fatores identificado na AFE e o modelo de seis fatores proposto pelos autores da versão original da ERA. De forma a avaliar a adequabilidade dos modelos aos dados da amostra, é necessária a análise de diversos índices de ajustamento (Byrne, 2010). Tendo em conta os critérios referidos por Marôco (2010), foram considerados os seguintes índices: o $\chi^{2}$ (o nível de significação associado deverá ser $>.05$ ), a razão entre o $\chi^{2}$ e os graus de liberdade $\left(\chi^{2} / g . l\right.$; cujo valor deverá situar-se entre 2 e 5), o Comparative Fit Index (CFI; este valor deverá ser $\geq .90$ ) e o Root Mean Square Error of Approximation (RMSEA; este valor deverá ser $<.10$ ).

O Quadro 6 apresenta os resultados da AFC. Tendo em consideração os critérios referidos, os valores do modelo unidimensional não corresponderam ao desejável. Os modelos de cinco (resultante da AFE) e seis fatores (estrutura original) apresentaram indicadores de ajustamento satisfatórios, com exceção do valor do $\chi^{2}$. O facto de o nível de significação deste índice não ser desejável pode decorrer 
da sua elevada sensibilidade ao tamanho da amostra (Marôco, 2010). Os modelos testados de cinco e seis fatores apresentaram, globalmente, índices de ajustamento satisfatórios, ainda que o valor do RMSEA se tenha situado no limiar dos valores não desejáveis. Face a estes resultados, a opção pela estrutura original na versão Portuguesa da ERA apresenta-se como adequada.

Quadro 6.

Índices de adequação dos modelos testados

\begin{tabular}{|c|c|c|c|c|}
\hline \multirow{2}{*}{ Modelo } & \multicolumn{4}{|c|}{ Índice } \\
\hline & $x^{2}$ & $\times 2 / g . l$. & $C F I$ & RMSEA (9O\% IC) \\
\hline Unidimensional & $2289.55(g . l .=495)$ & 4.63 & .643 & $.11(.10-.11)$ \\
\hline AFE - 5 fatores & $387.94($ g.l. $=94)$ & 4.12 & .897 & $.10(.09-.11)$ \\
\hline Original - 6 fatores & $364.94(g . l .=89)$ & 4.10 & .904 & $.10(.09-.11)$ \\
\hline
\end{tabular}

AFE - Análise Fatorial Exploratória; CFI - Comparative Fit Index; RMSEA - Root Mean Square Error of Approximation; IC - Intervalo de Confiança.

\section{Análises adicionais}

Considerando a estrutura original da ERA, foi novamente realizado o estudo da consistência interna, bem como analisadas as correlações entre os seis fatores. Assim, no que respeita à consistência interna, foi obtido um alfa de Cronbach de .94 para o total da escala. Relativamente aos seis fatores, obtiveram-se os valores seguintes: .38 (Estilo Estruturado), .72 (Competências Sociais), .75 (Planeamento do Futuro), .78 (Perceção do Self), .81 (Coesão Familiar) e .84 (Recursos Sociais).

As correlações entre os fatores variaram entre .49 (Estilo Estruturado e Coesão Familiar) e .82 (Recursos Sociais e Coesão Familiar). As correlações com o total da ERA variaram entre .68 (Estilo Estruturado) e .89 (Recursos Sociais). As correlações positivas e estatisticamente significativas (para $p<.001)$ entre os seis fatores e o total da ERA indicaram que estes avaliam o mesmo conceito, o que sustenta a validade de constructo da ERA.

Por fim, no âmbito dos estudos da validade dos grupos conhecidos, realizou-se a comparação dos resultados de resiliência nos três grupos de casais (população geral, diagnóstico de anomalia congénita de um filho, 
doença psiquiátrica de um dos elementos do casal). Os resultados mostraram a existência de diferenças estatisticamente significativas, Lambda de Wilks $=.65, F(6,297)=12.08, p<.001$. Concretamente, os participantes do grupo doença psiquiátrica apresentaram valores significativamente mais baixos em todas as características de resiliência, sendo os efeitos mais pronunciados na dimensão Coesão Familiar, $F(2,301)=67.71, p<$ .001. Não se registaram diferenças significativas entre os restantes dois grupos. Também no total da ERA, os participantes do grupo doença psiquiátrica reportaram valores significativamente mais baixos de resiliência, $F(2,301)=56.58, p<.001$.

\section{Aplicação}

\section{Como aplicar, cotar e interpretar?}

Como indicado no nome, a ERA poderá ser aplicada a indivíduos adultos, quer da população geral (amostras não clínicas) quer em amostras clínicas, não havendo restrição do contexto de aplicação, isto é, pode ser aplicada a doentes (em contexto de doença física e mental), a membros significativos da sua rede pessoal (e.g., parceiros(as), cuidadores), a indivíduos em situação de adversidade económico-social (e.g., desemprego, pobreza) ou em outras situações de risco e adversidade (e.g., acontecimentos traumáticos, divórcio).

A ERA foi desenvolvida como medida de auto-resposta. No entanto, em determinadas situações (e.g., dificuldades físicas ou menor nível de instrução do respondente) em que não é possível optar por esta metodologia, a sua aplicação pode ser assistida pelo entrevistador (leitura das instruções; explicação da forma de preenchimento do questionário e esclarecimento de dúvidas) ou mesmo administrado pelo entrevistador. Nesta última situação, o entrevistador deve ler as instruções, as perguntas, os descritores da escala de resposta (os dois pólos extremos de resposta) e assinalar no questionário a resposta dada pelo inquirido, tendo o cuidado de não alterar a formulação original dos itens. Quando aplicado como medida de auto-resposta, pode ser feita aplicação individual ou coletiva e o tempo do seu preenchimento é, geralmente, de cerca de 10 minutos. 
A cotação da ERA pode ser feita manualmente ou mediante recurso a uma sintaxe para o programa Statistical Package for the Social Sciences (IBM SPSS) que é sempre disponibilizada pelos autores da versão Portuguesa na altura do pedido de utilização. Cada pergunta é cotada de 1 a 7, no entanto, 17 dos 33 itens devem ser invertidos antes do seu uso nos cálculos finais, para que as pontuações mais elevadas correspondam a maiores níveis de resiliência. No Quadro 7 encontram-se listados os fatores e respectivos itens, com indicação dos itens a inverter.

O cálculo dos fatores que compõem a ERA, para uma análise de informação mais específica, pode ser feito de duas formas distintas: ou calculando a média dos resultados dos fatores (e total) ou através do somatório total de todos os indicadores. A primeira modalidade realiza-se somando os resultados nos itens de cada fator e dividindo esse valor pelo número total de itens. Por exemplo, para a Coesão Familiar (CF) são somados os seis itens do fator (após inversão) e divide-se o total por seis $\left[\mathrm{CF}=\left(\mathrm{ERA} \_04+\right.\right.$ ERA_10 + ERA_16 + ERA_22 + ERA_27 + ERA_31 $\left.) / 6\right]$.

Caso se opte pelos resultados totais, o procedimento consiste basicamente em somar os resultados nos itens de cada fator, após inversão dos respetivos itens. Por exemplo, para o fator Planeamento do Futuro (PF) são somados os quatro itens do fator (após inversão) [PF = ERA_02 + ERA_08 + ERA_14 + ERA_20]. Neste procedimento, o resultado total da ERA deverá variar entre 33 e 231.

Quadro 7.

Fatores e itens da ERA

\begin{tabular}{ll}
\hline Fatores & \multicolumn{1}{c}{ Itens } \\
\hline Perceção do Self (PS) & $1,7 \mathrm{i}, 13,19 \mathrm{i}, 25,29 \mathrm{i}$ \\
Planeamento do Futuro (PF) & $2,8 \mathrm{i}, 14 \mathrm{i}, 20$ \\
Competências Sociais (CS) & $3 \mathrm{i}, 9,15 \mathrm{i}, 21,26 \mathrm{i}, 30$ \\
Estilo Estruturado (EE) & $6 \mathrm{i}, 12,18 \mathrm{i}, 24$ \\
Coesão Familiar (CF) & $4,10 \mathrm{i}, 16,22 \mathrm{i}, 27,31 \mathrm{i}$ \\
Recursos Sociais (RS) & $5,11 \mathrm{i}, 17,23 \mathrm{i}, 28 \mathrm{i}, 32,33 \mathrm{i}$ \\
\hline
\end{tabular}

Nota: Os itens invertidos foram assinalados com a letra i.

De forma geral, a interpretação dos resultados da ERA é feita de forma linear a partir dos resultados obtidos nos diferentes fatores. Um resultado mais elevado corresponde a melhores níveis de resiliência. 
Dada a natureza multidimensional do conceito de resiliência que se encontra subjacente a este instrumento, os resultados podem ser analisados em função das pontuações obtidas nos seis fatores da ERA, mas também a partir da pontuação total da escala. Num contexto clínico, é possível uma leitura mais qualitativa dos resultados. Este procedimento pode ser particularmente adequado para identificação dos potenciais recursos protetores dos indivíduos que requerem maior atenção clínica e, especificamente, as competências e recursos que podem ser desenvolvidos.

\section{Vantagens, limitações e estudos futuros}

O presente capítulo teve como principal objetivo contribuir para a validação da versão em Português Europeu da Escala de Resiliência para Adultos. Em termos globais, a ERA revelou níveis satisfatórios de fidedignidade, tanto ao nível da consistência interna, como da estabilidade temporal. A AFC permitiu confirmar a estrutura original de seis fatores da ERA. Os resultados dos estudos de validade são também adequados, o que atesta a sua utilização em Portugal.

Os fatores da ERA apresentaram, em termos globais, valores aceitáveis de consistência interna, satisfazendo os critérios propostos por Pasquali (2003), segundo os quais é aceitável um valor de alfa superior a .70. De assinalar, porém, a baixa consistência interna do fator Estilo Estruturado. Por um lado, trata-se de um fator composto por um menor número de itens (4), o que pode contribuir para essa baixa consistência interna. Adicionalmente, poderá ter a ver com o tipo de escala de resposta utilizada, dado que tem sido assinalado que uma consequência de transformar a escala de resposta num formato de diferencial semântico é a redução da consistência interna (Friborg, Martinussen, \& Rosenvinge, 2006). Por outro lado, é um fator que tem apresentado também baixa consistência interna em diversos estudos com a ERA (Friborg et al., 2003, 2005; Hjemdal, Friborg, Stiles, Rosenvinge, \& Martinussen, 2006; Hjemdal et al., 2009, 2011). Tal como sugerido no estudo de validação belga (Hjemdal et al., 2011), a utilização deste fator deve ser feita com 
cuidado ou, em alternativa, a remoção completa deste fator (e, deste modo, reduzindo a escala de 33 para 29 itens) poderá ser uma proposta útil para melhorar o desempenho psicométrico desta escala. Estudos futuros serão necessários para identificar a melhor solução.

No que diz respeito à validade de constructo, adicionalmente à confirmação do modelo original de seis fatores, o padrão de correlações entre os fatores é coincidente com o objetivo dos autores originais, que sugere que estas dimensões avaliam diferentes aspetos positivos do conceito de resiliência (Friborg et al., 2003) e é consistente com outras versões da ERA (Hjemdal et al., 2011; Jowkar et al., 2010). Os estudos de associação dos fatores da ERA com as dimensões de resiliência avaliadas pela CD-RISC atestam a validade convergente da ERA. Os estudos de validade de grupos conhecidos, ao indicar a existência de diferenças significativas entre os participantes com doença psiquiátrica de um dos membros do casal, comparativamente aos casais da população geral e pais de crianças com diagnóstico de anomalia congénita, certificam o potencial discriminativo da ERA e corroboram a literatura que sugere que as pessoas com problemas psiquiátricos reportam menos fatores protetores nos seus contextos que aliviem o stress e previnam a inadaptação (Friborg et al., 2003).

Em acréscimo, as correlações com outros instrumentos, designadamente de perceção de auto-eficácia e da qualidade de vida contribuem também para certificar a relevância das características de resiliência no âmbito dos constructos positivos. Com efeito, considera-se que a ERA avalia um conceito importante, a resiliência, além de integrar os aspetos sociais e familiares, indicadores que são centrais neste conceito e não são contemplados em outras medidas de avaliação da resiliência (e.g., CD-RISC; Resilience Scale). Este constructo, enquadrando-se na linha de avaliação das dimensões positivas do funcionamento humano, contribui para a inversão da tendência para avaliar a adaptação e funcionamento humanos pelos seus resultados negativos.

Face ao exposto, os dados relativos à precisão e validade da versão da ERA aqui descrita asseguram o seu bom desempenho psicométrico, tornando-se uma medida multidimensional adequada para avaliação dos fatores de resiliência, cuja aplicação poderá ser estendida a diferentes 
contextos, quer clínicos quer de investigação. Nesta linha, avaliar os fatores de resiliência (que se podem entender como fatores protetores) a diferentes níveis, quer intrapessoais (competências pessoais/sociais) quer interpessoais (recursos familiares/sociais) pode ser útil para melhor conhecer como os diferentes níveis dos fatores protetores interagem com os fatores de stress e o risco.

As características de resiliência têm sido vistas como sendo mais estáveis ao longo do tempo do que, por exemplo, a sintomatologia psicopatológica (Friborg et al., 2003). Por este motivo, a avaliação das dimensões de resiliência pode ter um importante valor prognóstico dos resultados de intervenções terapêuticas, bem como possibilitar uma melhor previsão de recaídas e das competências dos doentes para lidar com dificuldades atuais ou futuras. Em contextos clínicos, pode também ser muito útil para identificar os recursos e potenciais fatores de proteção que podem ser direcionados para melhorar as competências existentes, promover trajetórias de ajustamento saudável e fomentar a adaptação resiliente, i.e., prevenindo a inadaptação e/ou perturbação. Em síntese, usar a resiliência como guia de literatura pode ser importante para orientar estratégias de avaliação e planeamento de intervenções, porque ao ter em conta os aspetos do contexto ecológico do indivíduo pode permitir aos profissionais identificar melhor os fatores e sistemas, tanto formais como informais, que podem ser incorporados no processo de intervenção (Tedeschi \& Kilmer, 2005).

Este estudo não está isento de limitações. Em primeiro lugar, as impostas pela amostragem por conveniência devem ser consideradas na generalização dos resultados. Algumas características da amostra de validação não possibilitam um grau de representatividade que permita a extrapolação das conclusões para a população (e.g., a idade média de 35.20 anos é inferior à média da população adulta portuguesa; as habilitações literárias médias da amostra são superiores às da população portuguesa, nomeadamente pelo facto de uma parte da amostra ter sido recolhida junto de estudantes universitários). Também as características da segunda amostra devem ser tidas em conta, principalmente porque composta por casais, em situação de emprego e predominantemente a viver em meio urbano, o que poderá 
também ter condicionado a representatividade da amostra. Apesar de no presente estudo termos usado participantes em diferentes contextos de risco ou adversidade, o tamanho da amostra não permitiu realizar uma AFC multi-grupos, de forma a testar se a solução fatorial da ERA é replicada em populações normativas e de risco. O desenho transversal deste estudo pode ser considerado uma limitação, porém, tendo em conta que o padrão de associações está em linha com aplicações anteriores da ERA, podemos ter alguma confiança nos resultados obtidos.

Tendo em consideração que os estudos existentes com a ERA têm sido realizados fundamentalmente em populações normativas, estudos em populações mais diversas, e particularmente em diferentes contextos de risco ou adversidade serão desejáveis, sobretudo para avaliar se estes fatores protetores são transversais a diferentes contextos. Nestes, será particularmente relevante realizar estudos de desenho longitudinal, que considerem a resiliência como um processo dinâmico e não apenas como um conjunto de indicadores de competências e adaptação. Para que estes estudos possam ser efetivos, será importante, do ponto de vista da avaliação, continuar a investir em estudos que possam confirmar a robustez psicométrica das medidas existentes, nomeadamente a ERA.

\section{Bibliografia}

Ahern, N. R., Kiehl, E. M., Sole, M. L., \& Byers, J. (2006). A review of instruments measuring resilience. Issues in Comprehensive Pediatric Nursing, 29, 103-125 doi:10.1080/ 01460860600677643

Albuquerque, S., Pereira, M., Fonseca, A., \& Canavarro, M. C. (2012). Impacto familiar e ajustamento de pais de crianças com diagnóstico de anomalia congénita: Influência dos determinantes da criança. Revista de Psiquiatria Clínica, 39, 136-141. doi:10.1590/ S0101-60832012000400004

Alves, S., Pereira, M., Janeiro, C., Narciso, I., \& Canavarro, M. C. (2014). O papel do ajustamento diádico na sintomatologia psicopatológica e qualidade de vida de doentes com perturbação psiquiátrica e dos parceiros saudáveis. Análise Psicológica, 32(3), 323-339. doi:10.14417/ap.830

Becvar, D. (2007). Families that flourish: Facilitating resilience in clinical practice. New York, NY: W. W. Norton \& Company, Inc.

Bock, J., \& Kremen, A. M. (1996). IQ and ego-resiliency: Conceptual and empirical connections and separateness. Journal of Personality and Social Psychology, 70, 349-361. doi:10.1037/0022-3514.70.2.349 
Bonanno, G. A. (2004). Loss, trauma and human resilience: Have we underestimated the human capacity to thrive after extremely aversive events? American Psychologist, 59, 20-28. doi:10.1037/0003-066X.59.1.20

Byrne, B. (2010). Structural equation modeling with AMOS: Basic concepts, applications, and programming (2nd ed.). New York: Routledge.

Campbell-Sills, L., \& Stein M. B. (2007). Psychometric analysis and refinement of the ConnorDavidson Resilience Scale (CD-RISC): Validation of a 10-item measure of resilience. Journal of Traumatic Stress, 20, 1019-1028. doi:10.1002/jts.20271

Cicchetti, D., \& Garmezy, N. (1993). Prospects and promises in the study of resilience. Development and Psychopathology, 5, 497-502. doi:10.1017/S0954579400006118

Cohen, S., Kamarck, T., \& Mermelstein, R. (1983). A global measure of perceived stress. Journal of Health and Social Behavior, 24, 385-396. doi:10.2307/2136404

Connor, K. M., \& Davidson, J. T. (2003). Development of a new resilience scale: The ConnorDavidson Resilience Scale (CD-RISC). Depression and Anxiety, 18, 76-82. doi:10.1002/ da.10113

Curtis, W. J., \& Cicchetti, D. (2007). Emotion and resilience: A multilevel investigation of hemispheric electroencephalogram asymmetry and emotion regulation in maltreated and nonmaltreated children. Development and Psychopathology, 19, 811-840. doi:10.1017/ S0954579407000405

Davydov, D., Stewart, R., Ritchie, K., \& Chaudieu, I. (2010). Resilience and mental health. Clinical Psychology Review, 30, 479-495. doi:10.1016/j.cpr.2010.03.003

Friborg, O., Barlaug, D., Martinussen, M., Rosenvinge, J., \& Hjemdal, O. (2005). Resilience in relation to personality and intelligence. International Journal of Methods in Psychiatric Research, 14, 29-42. doi:10.1002/mpr.15

Friborg, O., Hjemdal, O., Rosenvinge, J., \& Martinussen, M. (2003). A new rating scale for adult resilience: What are the central protective resources behind healthy adjustment? International Journal of Methods in Psychiatric Research, 12, 65-76. doi:10.1002/mpr.143

Friborg, O., Hjemdal, O., Rosenvinge, J. H., Martinussen, M., Aslaksen, P. M., \& Flaten, M. A. (2006). Resilience as a moderator of pain and stress. Journal of Psychosomatic Research, 61, 213-219. doi:10.1016/j.jpsychores.2005.12.007

Friborg, O., Martinussen, M., \& Rosenvinge, J. H. (2006). Likert-based versus semantic differential-based scorings of positive psychological constructs: A psychometric comparison of two versions of a scale measuring resilience. Personality and Individual Differences, 40, 873-884. doi:10.1016/j.paid.2005.08.015

Gurgel, L. G., Plentz, R. D. M., Joly, M. C., \& Reppold, C. T. (2013). Avaliação da resiliência em adultos e idosos: Revisão de instrumentos. Estudos de Psicologia (Campinas), 30 , 487-496. doi:10.1590/S0103-166X2013000400002

Hawking, S. W. (1988). A brief history of time: From the Big Bang to Black Holes. New York: Bantam.

Hjemdal, O., Friborg, O., Braun, S., Kempenaers, C., Linkowski, P., \& Fossion, P. (2011). The resilience scale for adults: Construct validity and measurement in a Belgian sample. International Journal of Testing, 11, 53-70. doi:10.1080/15305058.2010.508570

Hjemdal, O., Friborg, O., Martinussen, M., \& Rosenvinge, J.H. (2001). Preliminary results from the development and validation of a Norwegian scale for measuring adult resilience. Journal of the Norwegian Psychology Association, 38, 310-317.

Hjemdal, O., Friborg, O., Stiles, T. C., Martinussen, M., \& Rosenvinge, J. H. (2006). A new rating scale for adolescent resilience: Grasping the central protective resources behind healthy development. Measurement and Evaluation in Counseling and Development, 39, 84-96. 
Hjemdal, O., Friborg, O., Stiles, T. C., Rosenvinge, J. H., \& Martinussen, M. (2006). Resilience predicting psychiatric symptoms: A prospective study of protective factors and their role in adjustment to stressful life events. Clinical Psychology and Psychotherapy, 13, 194-201. doi:10.1002/cpp.488

Hjemdal, O., Roazzi, A., Dias, M. G., Roazzi, M., \& Vikan, A. (2009). Exploring the psychometric properties of the resilience scale for adults in a Brazilian sample. In D. Elizur, \& E. Yaniv (Orgs.), Facet new horizons in theory construction and data analysis (pp. 120138). Jerusalem: FTA.

Hoge, E. A., Austin, E. D., \& Pollack, M. H. (2007). Resilience: Research evidence and conceptual considerations for posttraumatic stress disorder. Depression and Anxiety, 24, 139-152. doi:10.1002/da.20175

Jowkar, B., Friborg, O., \& Hjemdal, O. (2010). Cross-cultural validation of the Resilience Scale for Adults (RSA) in Iran. Scandinavian Journal of Psychology, 51, 418-425. doi:10.1111/j.1467-9450.2009.00794.x

Luthar, S., Cicchetti, D., \& Becker, B. (2000). The construct of resilience: A critical evaluation and guidelines for future work. Child Development, 71, 543-562. doi:10.1111/14678624.00164

Marôco, J. (2010). Análise de equações estruturais: Fundamentos teóricos, software \& aplicações. Pêro Pinheiro: ReportNumber.

Masten, A. S. (2007). Resilience in developing systems: Progress and promise as the fourth wave rises. Development and Psychopathology, 19, 921-930. doi:10.1017/ S0954579407000442

Masten, A. S., \& Coatsworth, J. D. (1998). The development of competence in favorable and unfavorable environments: Lessons from successful children. American Psychologist, 53, 205-220. doi:10.1037/0003-066X.53.2.205

Mota-Cardoso, R., Araújo, A., Ramos, R. C., Gonçalves, G., \& Ramos, M. (2002). O stress dos professores portugueses: Estudo IPSSO 2000. Porto: Porto Editora.

Oshio, A., Kaneko, H., Nagamine, S., \& Nakaya, M. (2003). Construct validity of the Adolescent Resilience Scale. Psychological Reports, 93, 1217-1222. doi:10.2466/pr0.2003.93.3f.1217

Pasquali, L. (2003). Psicometria - Teoria dos testes na psicologia e educação. Petróplois: Editora Vozes.

Pereira, M., Cardoso, M., Alves, S., Narciso, I., \& Canavarro, M. C. (2013). Estudos preliminares das características psicométricas da Escala de Resiliência para Adultos (ERA). In A. Pereira, M. Calheiros, P. Vagos, I. Direito, S. Monteiro, C. F. Silva, \& A. Allen Gomes (Eds.), Livro de atas do VIII Simpósio Nacional de Investigação em Psicologia (pp. 93103). Aveiro: Associação Portuguesa de Psicologia.

Prince-Embury, S. (2008). The Resiliency Scales for Children and Adolescents, psychological symptoms and clinical status in adolescents. Canadian Journal of School Psychology. 23, 41-56. doi:10.1177/0829573508316592

Reppold, C. T., Mayer, J. C, Almeida, L. S., \& Hutz, C. S. (2012). Avaliação da resiliência: Controvérsia em torno do uso das escalas. Psicologia: Reflexão e Crítica, 25, 248-255. doi:10.1590/S0102-79722012000200006

Rutter, M. (1990). Psychological resilience and protective mechanisms. In J. Rolf, A. S. Masten, D. Cicchetti, K. Nuecherterlein, \& S. Weintraub (Eds.), Risk and protective factors in the developmental psychopathology (pp. 181-214). Cambridge: Cambridge University Press.

Schaap, I. A., Galen, F. M., Ruijter, A. M., \& Smeets, E. C. (2009). Resilience, the article: The balance between awareness and fear. Retirado de www.impact-kenniscentrum.nl/ download/file1163776605.pdf 
Schwarzer, R., \& Jerusalem, M. (1995). Generalized Self-Efficacy Scale. In J. Weinman, S. Wright, \& M. Johnston (Eds.), Measures in health psychology: A user's portfolio (pp. 3537). Windsor: NFER-NELSON.

Sinclair, V. G., \& Wallston, K. A. (2004). The development and psychometric evaluation of the Brief Resilient Coping Scale. Assessment, 11, 94-101. doi:10.1177/1073191103258144

Smith, B. W., Dalen, J., Wiggins, K., Tooley, E., Christopher, P., \& Bernard, J. (2008). The brief resilience scale: Assessing the ability to bounce back. International Journal of Behavioural Medicine, 15, 194-200. doi:10.1080/10705500802222972

Smith-Osborne, A., \& Bolton, K. W. (2013). Assessing resilience: A review of measures across the life course. Journal of Evidence-Based Social Work, 10, 111-126. doi:10.1080/ 15433714.2011 .597305

Tedeschi, R. G., \& Kilmer, R. P. (2005). Assessing strengths, resilience, and growth to guide clinical interventions. Professional Psychology: Research and Practice, 36, 230-237. doi:10.1037/0735-7028.36.3.230

Vaishnavi, S., Connor, K., \& Davidson, J. T. (2007). An abbreviated version of the ConnorDavidson Resilience Scale (CD-RISC), the CD-RISC2: Psychometric properties and applications in psychopharmacological trials. Psychiatry Research, 152, 293-297. doi:10.1016/j.psychres.2007.01.006

Vaz Serra, A., Canavarro, M. C., Simões, M., Pereira, M., Gameiro, S., Quartilho, M. J., ... Paredes, T. (2006). Estudos psicométricos do instrumento de avaliação da qualidade de vida da Organização Mundial de Saúde (WHOQOL-Bref) para Português de Portugal. Psiquiatria Clínica, 27, 41-49.

Wagnild, G. M. \& Young, H. M. (1993). Development and psychometric evaluation of the Resilience Scale. Journal of Nursing Measurement, 1, 165-178.

Walsh, F. (2003). Family resilience: Framework for clinical practice. Family Process, 42, 1-18. doi:10.1111/j.1545-5300.2003.00001.x

Windle, G., Bennett, K. M., \& Noyes, J. (2011). A methodological review of resilience measurement scales. Health and Quality of Life Outcomes, 9:8. Retirado de http://www. hqlo.com/content/9/1/8

WHOQOL Group (1998). Development of World Health Organization WHOQOL-BREF quality of life assessment. Psychological Medicine, 28, 551-558. doi:10.1017/S0033291798006667 